\title{
REDESIGN OF IMPRESSION TRAYS USING REVERSE ENGINEERING AND FINITE ELEMENT ANALYSIS
}

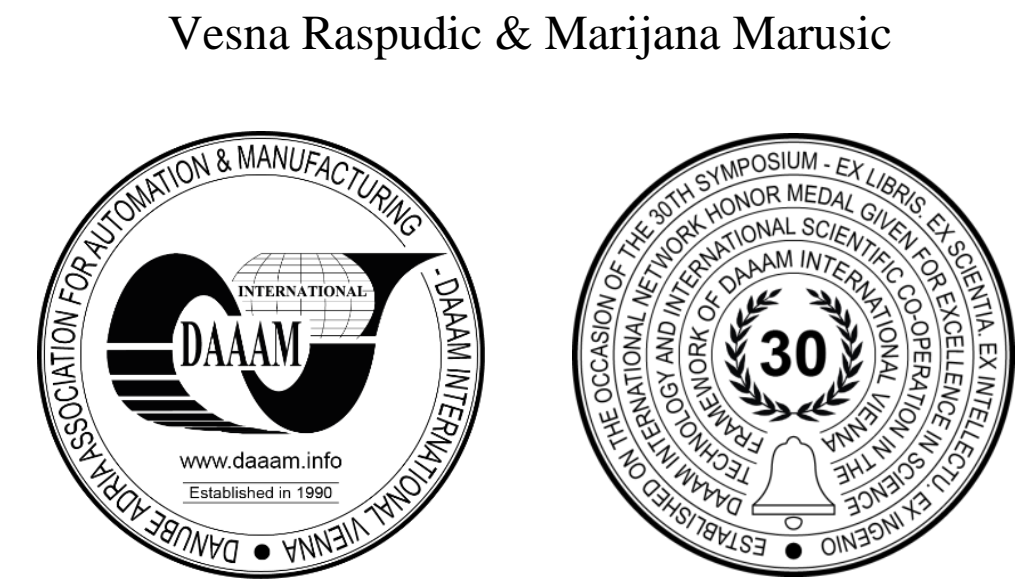

This Publication has to be referred as: Raspudic, V[esna] \& Marusic, M[arijana] (2021). Redesign of Impression Trays using Reverse Engineering and Finite Element Analysis, Proceedings of the 32nd DAAAM International Symposium, pp.0333-0338, B. Katalinic (Ed.), Published by DAAAM International, ISBN 978-3-902734-33-4, ISSN 1726-9679, Vienna, Austria

DOI: $10.2507 / 32$ nd.daaam.proceedings.049

\begin{abstract}
The reverse engineering technologies facilitate development of new or redesign of existing products, which has been presented in this paper on an example of dental impression trays redesign. There are different types of trays available today, in order to ensure proper retention, stability and support in the impression taking procedure. Disposable lower and upper trays have been used as the initial samples for 3D scanning, which was performed by the GOM Atos Compact Scan $8 \mathrm{M}$ optical scanner. The CAD models were build using Geomagic and SolidWorks, while the redesign consisted of introducing new perforations in order to allow the impression material to expand through the holes and so be held securely in place. Finite element analysis has been used to investigate the effect of introducing these design changes to structural rigidity. Due to the need to consult a prosthetist about the acceptability of the redesign, the models have been printed by the 3D printer Zortrax M200 Plus.
\end{abstract}

Keywords: Dental impression tray; Reverse Engineering; FEA; Rapid Prototyping

\section{Introduction}

The development of CAD/CAM/CAE has greatly automated and facilitated a range of activities related to design, simulation and optimization processes in a number of areas of industrial application. There are two different approaches to the engineering design process, and these are traditional and reverse engineering. In traditional engineering, product design is generally done by forward design, which proposes conceptual ideas according to the function and use of the product, models it and finally processes and shapes it by technical means. The design model is mostly done by the designer on CAD software, and this method often has a long cycle time and low efficiency. In contrast to this approach, reverse engineering (RE) is the process of creating a CAD model from a physical part or prototype. RE techniques convert point cloud data, obtained by digitization by contact or noncontact scanning, into CAD models either in NURBS or STL format. These CAD models can subsequently be used to fabricate using either conventional material removal methods or material incremental methods, i.e. rapid prototyping (RP). 
Engineering modelling often combines advances of both of these methods, which is a hybrid process of reverse engineering and forward modelling, to shorten the product design cycle, improve efficiency and meet the fast-paced changes of the modern market.

The technology advances of CAD/CAM/CEA have also made a significant impact in medical fields, providing especially many benefits for restorative dentistry [1], [2]. In prosthodontics, the digital method of obtaining dental impressions has proven to be more efficient than conventional one when assessing the total treatment time and patient comfort [3], [4]. Nevertheless, there are studies showing that digital scanning systems are not superior to conventional methods in comparing fidelity, accuracy and detail reproduction [5]. Also, the high cost of digital systems often makes their use difficult in clinical practice, so the conventional impression method is still a standard procedure in many dental practices.

The purpose of the dental impression is to copy the patient's intraoral situation, transforming it into a model. Obtaining a model of good quality and true to the original is extremely important for the success of the restoration. In conventional impression methods, different types of materials and impression techniques have been used over the years to achieve the desired accuracy. Independent studies of the quality and accuracy of impressions for crown and bridge works have shown that about $40 \%$ to $50 \%$ of impressions were not good enough to reliably obtain a satisfactory, well-fitting restoration for the first time [6].

Looking at the reasons for the impression defects, the researchers found that one of the most interesting factors is the use of an appropriate impression tray. The tray is required to give rigid support to the impression material and to facilitate its introduction into the mouth. They can be categorized as custom trays made specifically for a patient or stock trays that are available in a variety of sizes from the manufacturer. Custom trays are used to make a final impression, while stock trays are used for an initial or preliminary impression.

In this paper a procedure based on reverse engineering is implemented, where 3D scanning has been used for creating CAD models of disposable perforated lower and upper impression trays. Based on them, new models with additional perforations were designed using Geomagic and SolidWorks software. Static analysis in SolidWorks Simulation has been used to predict the physical behaviour of products by virtually testing models.

\section{Rigidity of disposable stock trays}

Stock trays can be subcategorized into two general types: metal stock trays and disposable plastic stock trays. It is important that the impression material remains attached to the tray during the impression. Partial detachment can cause gross distortions of the impression that may remain undetected and will almost certainly lead to ill-fitting appliances or restorations. Manufacturers of impression materials often supply tray adhesives that are used to enhance bonding. Additional retention is achieved by using perforated trays. The impression material flows through the perforations allowing a more even distribution of the impression material and giving a mechanical lock to the tray.

There is a strong preference between metal and plastic trays for disposable plastic ones, as they do not need to be returned from the laboratory and do not have to be cleaned and disinfected after use. An important question, however, is whether there is a significant difference in the precision of impressions made with metal and plastic stock trays, as they should be rigid enough to withstand the forces generated during impression procedures without permanent deformation. Studies have shown that most disposable plastic trays are too flexible to ensure accuracy, especially when the two-stage putty/wash silicone impression material is used [6]. The procedure requires light-bodied material to be added to the putty silicone, and when this occurs stresses can be induced in the putty. If a stock tray made of flexible plastic is used, these stresses can deform the tray. The critical area for tray stiffness is bucco-lingually. During seating, the highly viscous material will expand disposable tray (Fig. 1a). If the tray deforms, it will spring back to a smaller bucco-lingual width when removed from the mouth (Fig. 1b), resulting in castings which are then too narrow along this dimension.

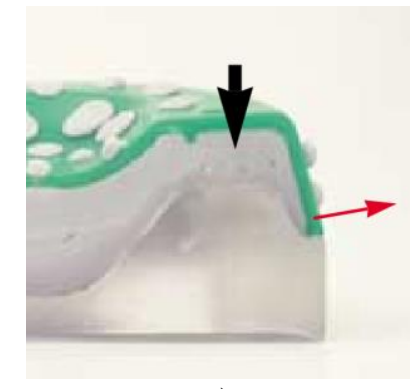

a)

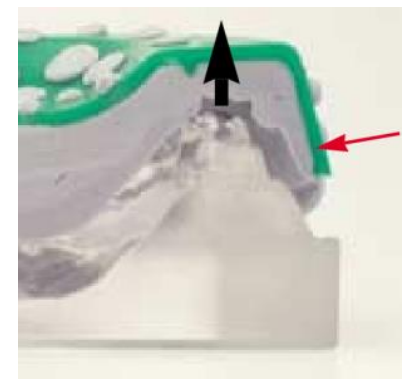

b)

Fig. 1. Bucco-lingually deformation of disposable tray [6]

Dimensional distortion of the impression is also more likely when a flexible tray is used, if excessive pressure is applied during impression making. An adequate impression tray should be rigid, i.e. flexion and torsion resistant, in order to resist the forces generated during impression procedures without permanent deformation. In [7], the authors studied 
the cross-sectional and cross-arch changes of six disposable plastic trays and compared them with a metal stock tray. The tested metal tray did not produce any significant changes, while all related disposable plastic trays evaluated produced significant dimensional changes when measured without impression material, on seating and polymerization of the impression material, and on removal of the impression material.

\section{Generating CAD models based on RE}

The 3D scanning system includes the GOM Atos Compact Scan 8M optical scanner, a movable stand to which the 3D scanner is attached, a laptop, a rotary table, an optical cable that connects the 3D scanner to the computer and a power adapter (Fig. 2). The software used for 3D scanning was ATOS Professional 2020.

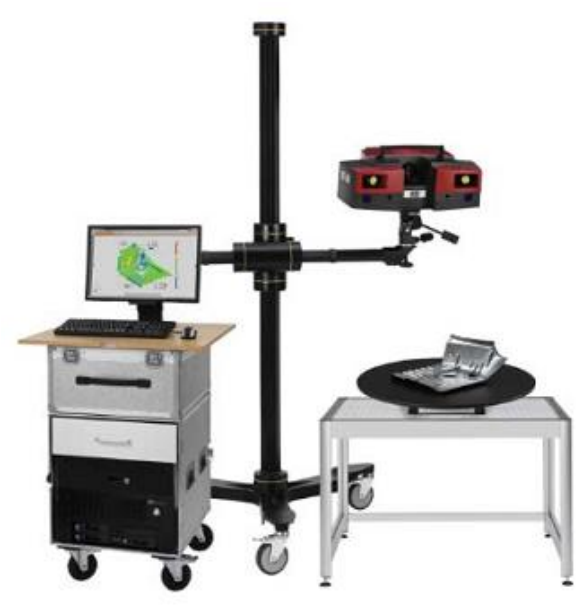

Fig. 2. The 3D scanning system [8]

GOM Atos Compact Scan $8 \mathrm{M}$ is a mobile 3D scanner that provides adaptability to different applications, part sizes and environments [8]. It is a type of coordinate measuring machine that uses a contactless blue light projector and two cameras that record line projections. On the scanner, there is a possibility of replacing the lenses on the cameras, which changes the measuring volume depending on the size of the object being scanned. In this case, the measuring volume 350 has been used. Before starting the scanning process, it is necessary to prepare the object. The first step begins with its cleaning, i.e. degreasing in case there are certain physical impurities. Reference point markers are then placed on its surface, which are of a specific shape (white dot on a black background) and diameter, depending on the measured volume. They must not be set near the edges of the scanning object. The 3D scanner automatically recognizes and encodes these markers, using them as reference points to properly align subsequent scans. The prepared object needs to be placed on a rotary table on which measuring points have been previously prepared and glued, which will later serve as a reference for separating the scanning object from the environment. The scanning object can be attached to a table to prevent it from moving and thus prevent the image quality from deteriorating.

It is also necessary to check that the distance between the sensor and the object is satisfactory, followed by setting the image saturation parameters. The angle of rotation of the rotary table, and thus the object itself, was set with a step of $72^{\circ}$ between two consecutive images, and if necessary, two more images could be added to further fill the gaps created in the scan. After scanning one side of the object, the same procedure is performed for the other side.

The obtained scan results are further processed in the GOM software, which includes the Transform Measurement Series option where the software can recognize points to be connected or they can be manually selected. When merging multiple scanned sides of an object, overlapping scanned parts and points that potentially deviate from the geometry are automatically removed. The connection is made by polygonization of points, which gives a mesh of polygonized triangles.

Before solid modelling, the mesh is further processed. This primarily refers to the alignment of the workpiece and the filtering of defects that may occur on the surface (roughness, glare, etc.) or may be systemic, which are manifested in the appearance of spikes due to non-smoothing of the surface. The final step is to export the mesh in STL format and import it into Geomagic, which is a reverse engineering software toolset that allows importing scan data directly in SolidWorks. Using Geomagic ability to extract reference geometries and cross-sections directly from point cloud data, multiple crosssectional curves associated with the original models were created. They were used as a basis for defining profile and guide curves for the standard set of SolidWorks tools for creating 3D feature based, parametric models. Afterwards, deviation analysis was performed. Based on these CAD models, the redesigned impression trays were constructed on which posterior area became less rounded and new perforations were generated. They should allow the impression material to escape through them, which contributes to a more even material distribution and better retention.

The redesigned models have been printed by the 3D printer Zortrax M200 Plus, in order to consult a prosthetist on the acceptability of the redesign (Fig. 3). 


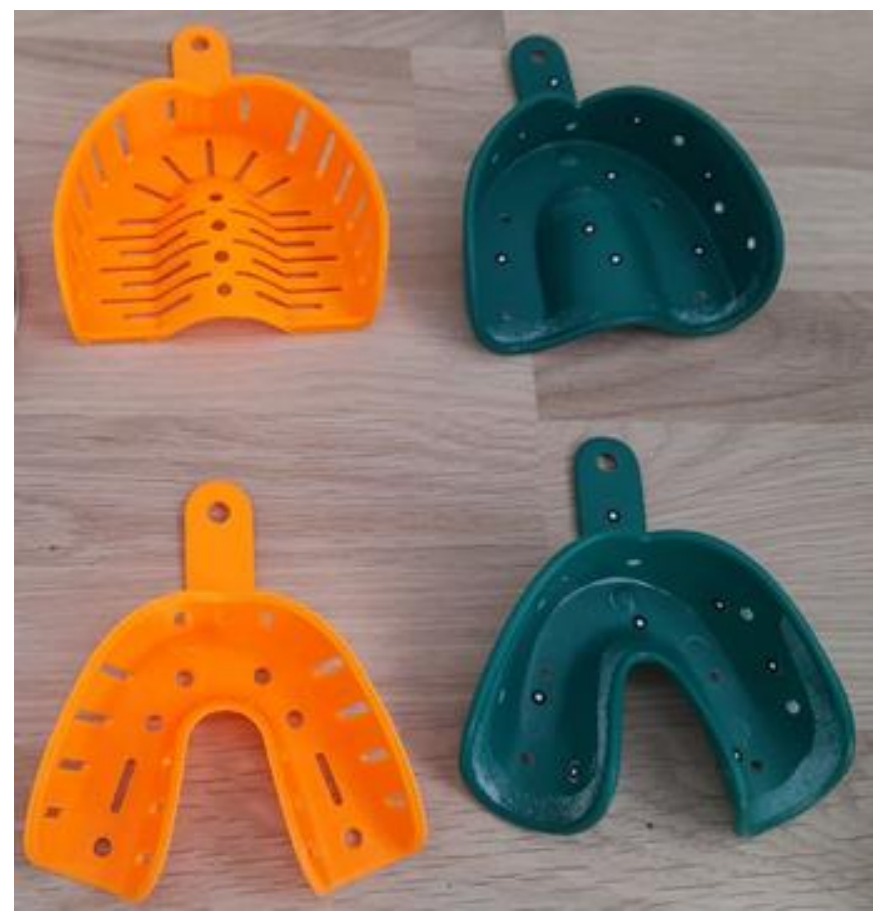

Fig. 3. The printed redesigned and original impression trays

\section{Finite element analysis and discussion of the results}

The CAD models obtained by the scanning process, Geomagic and SolidWorks software were used as a basis for creating FEM models in SolidWorks Simulation static study. The material used in this study was Polystyrene PS Medium/High Flow from SolidWorks material database. It was assumed to be homogenous, isotropic and to have linear elasticity, with elastic modulus E= $2280 \mathrm{~N} / \mathrm{mm} 2$ and Poisson's Ratio $v=0.387$.

The mesh was created using the second order tetrahedral elements, with mesh controls defined on geometry elements where a high stress concentration was expected.

The forces required to insert and remove an impression from mouth have been considered by some investigators [9]. It has been shown that they depend on the properties of the impression and tray materials, the tray design, the method used to seat and remove the completed impression, and the shape and consistency of the soft tissues. In [9], Sotiriou and Hobkirk examined forces exerted during in vivo recording of impressions of dentate maxillary arches. They used four different impression materials in metal and disposable plastic trays. The load rate and the maximum insertion and removal forces were measured with strain gauges mounted on the tray handles. When the impression tray was inserted and removed from the mouth, the tray was held with the thumb and the index finger of the right hand of the operator, and the necessary forces were applied only on the handle of the tray. In this study, a boundary condition was applied that prevents the nodes from moving on the surfaces of the handle on which it is held.

There are a number of studies that have investigated the pressure applied to the tissue during final impression making [10], [11]. These studies were mainly conducted in vitro and with custom impression trays. The results show that when impression material is placed in a tray and compressed, the highest pressure is located in the central region, and pressure gradually decreases in the direction of the periphery. This is due to the rheological properties of the impression material, as the internal pressure of the material decreases in the direction of the peripheral area where the pressure escapes [12].

Based on the results of the aforementioned studies, we made an assumption that the pressure acting on the tray during initial impression was distributed by the equation:

$$
p(x, y)=\frac{p_{0}}{L} x\left(1-\frac{y}{H}\right)
$$

where $p_{0}$ is the pressure acting in the most anterior region of the tray body; $L$ and $H$ are the length and height of the tray; $x$ and $y$ are coordinates relative to the local coordinate system with the origin placed at the most posterior point of the mid-sagittal line on the plane coinciding with the tray floor, as shown for the upper trays in Fig. 4. The equation (1) assumes linear increase in pressure from the posterior towards anterior part, as well as linear pressure decrease from the tray floor towards the tray flanges borders. The maximum value of pressure $p_{0}$ is estimated based on the resulting force on the tray handle, which corresponds to the average force records for plastic trays in [9]. The value used in this study was $p_{0}=5 \mathrm{kPa}$. 


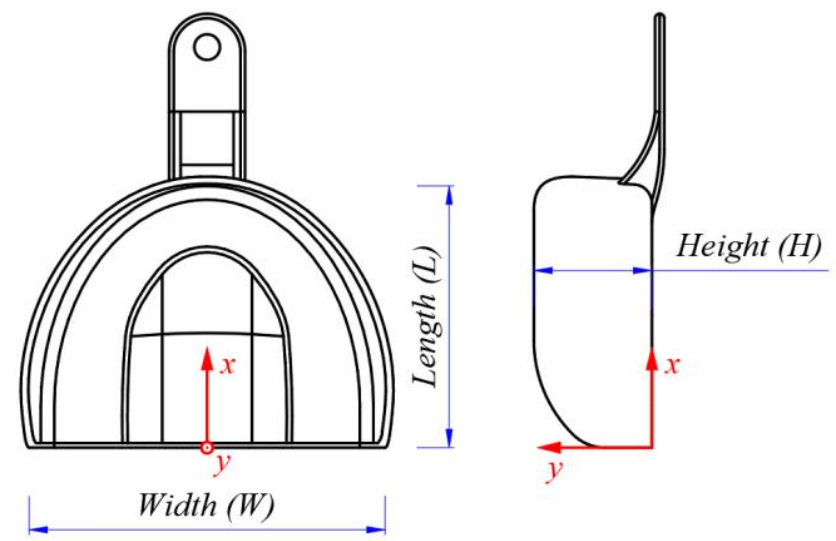

Fig. 4. Local coordinate system for the upper trays

In this study, a qualitative analysis of stress distribution using Von Mises criteria and principle stresses to measure the overall stresses has been performed. The stress distribution for all tray designs showed that for the assumed load model the highest stresses occurred on the tray handle, with values within the safe limits of the material used, which ensured the safe use of all designs.

Deformation plots are shown in Fig. 5-7. They are presented with undeformed models (in grey colour) superimposed on the deformed shapes, which helps to capture the deformed shape relative to the original shape. This type of display can also facilitate insight into which areas of the trays most deviate from the initial undeformed shape, and accordingly in which areas of the cast model deviations from the anatomical shape can be expected. The maximum deformations for the upper trays are in the vertical direction at the most posterior point of the mid-sagittal line (Fig. 5-6). The difference between these values for the original and redesigned tray is about $10 \mu \mathrm{m}$, and the same difference in deformation values is also present in their bucco-palatal direction. For the lower trays, maximal deformations are present at the posterior areas of the lingual flanges, with a value difference of about $20 \mu \mathrm{m}$ between the two tray configurations (Fig. 7).
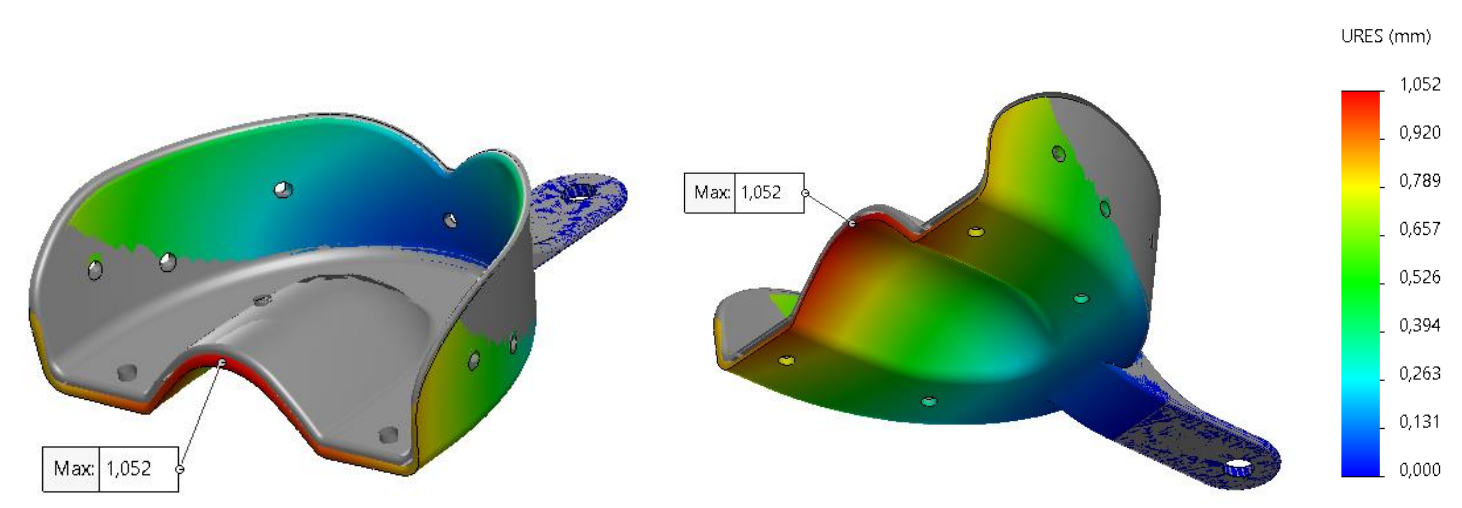

Fig. 5. Total deformation of the upper original tray
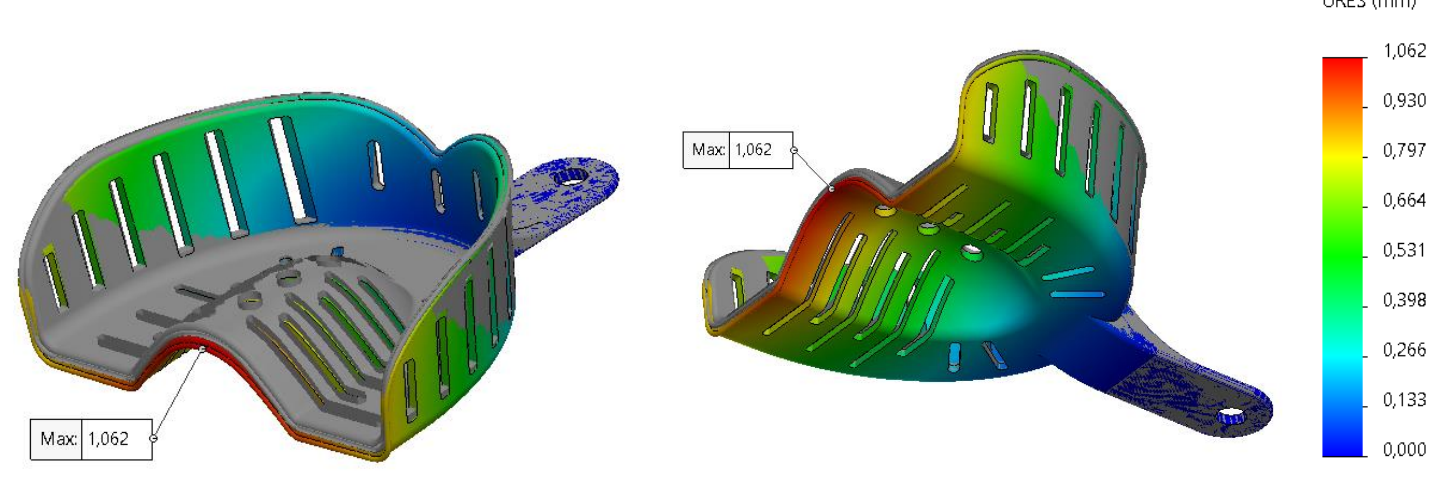

Fig. 6. Total deformation of the upper redesigned tray 

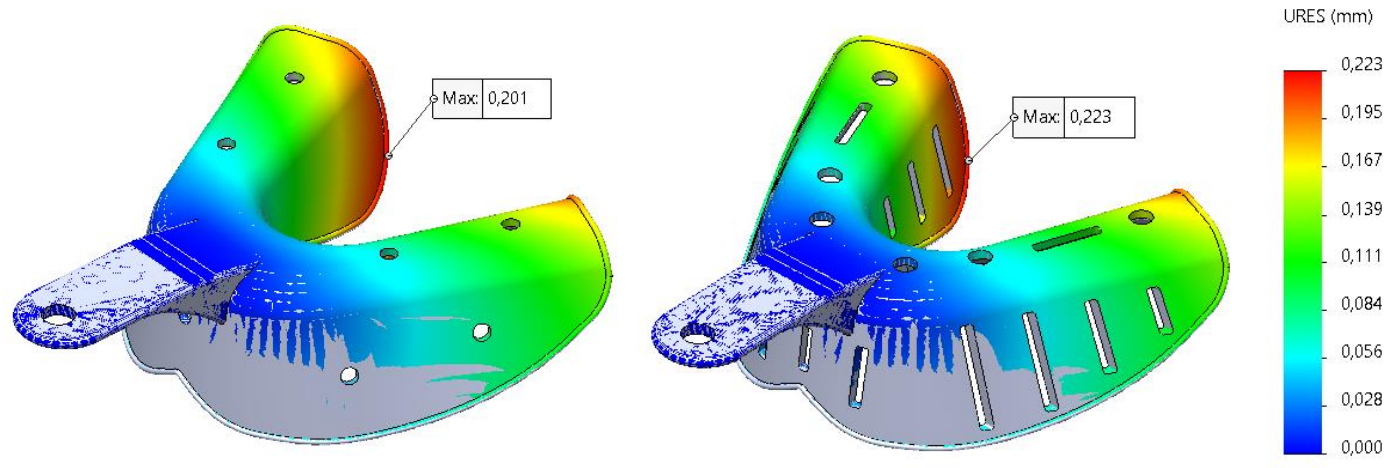

Fig. 7. Total deformation of the lower original and redesigned tray

\section{Conclusion}

RE can imitate and redesign products based on existing physical parts or prototypes, while RP can produce parts of a very complex geometric shape in a short time compared to traditional methods of producing spatial objects. In this paper, $\mathrm{RE}$ and RP have been used in order to redesign disposable upper and lower impression trays. Tray rigidity is an important parameter when taking impressions because even slightly flexing of the tray causes distortion of the impression. The FEA has been used to test the structural rigidity for the proposed load model to which the trays are exposed when taking impressions. Based on the results of the numerical studies, both tray configurations, the original and redesigned ones, have similar deformation pattern, with the displacement values of the redesigned models being higher because their rigidity decreases due to the additional perforations.

Further research into distortion of impressions done with the trays would provide data to define more suitable numerical models for obtaining values that may be closely correlated with values measured intraorally or with in vitro laboratory tests. Thus, the application of FEA creates an opportunity to recreate mechanical forces corresponding to the real clinical condition and to obtain stresses and strains whose direct measurement would be difficult if an adequate computational model was not available. This would enable the design to be optimized in terms of a more suitable perforation arrangement to achieve proper retention, stability and support in the impression taking procedure while maintaining maximum tray rigidity.

\section{References}

[1] Abduo, J.; Lyons, K. \& Bennamoun, M. (2014). Trends in computer-aided manufacturing in prosthodontics: A review of the available streams, International Journal of Dentistry, Volume 2014, Article ID 783948, http://dx.doi.org/10.1155/2014/783948

[2] Beuer, F.; Edelhoff, D. \& Schweiger, J. (2008). Digital dentistry: an overview of recent developments for CAD/CAM generated restorations, British Dental Journal, Vol. 204(9), pp. 505-511, DOI: 10.1038/sj.bdj.2008.350

[3] Lima, J., et al. (2019). Evaluation of the accuracy of conventional and digital methods of obtaining dental impressions, International Journal of Odontostomatology, Vol. 12(4), pp. 368-375, DOI: 10.4067/S0718381 X2018000400368

[4] Park, H.S. \& Shah, C. (2014). Development of high speed and high accuracy 3D dental intra oral scanner, Proceedings of the 25th International DAAAM Symposium "Intelligent Manufacturing \& Automation", 26-29th November 2014, Vienna, Austria, Procedia Engineering 100 ( 2015 ) 1174 - 1181

[5] Chandran, S., et al. (2019). Digital versus conventional impressions in dentistry: A systematic review, Journal of Clinical and Diagnostic Research, Vol. 13(4): ZE01-ZE06, DOI: 10.7860/JCDR/2019/38494.12756

[6] https://europe.gc.dental/sites/europe.gc.dental/files/products/downloads/coeimpressiontrays/manual/, The foundation of a good restoration. Impression Trays. Guide to correct tray selection, Accessed on: 2021-06-20

[7] Cho, G. \& Chee, W. (2004). Distortion of disposable plastic stock trays when used with putty vinyl polysiloxane impression materials, The Journal of Prosthetic Dentistry, Vol. 92(4), pp. 354-358, DOI: 10.1016/j.prosdent.2004.07.020

[8] https://www.gom.com/en/products/3d-scanning/, Industrial 3D scanning technology, Accessed on: 2021-06-15

[9] Sotiriou, M. \& Hobkirk, J. (1995). An in vivo investigation of seating and removal forces associated with recording impressions in dentate patients, The Journal of Prosthetic Dentistry, Vol. 74(5), pp. 455-462

[10] Chang, Y., et al. (2019). Influence of edentulous tray modification on the pressure distribution of the impression: An in vitro study, The International Journal of Prosthodontics, Vol. 32(3), pp. 278-280

[11] Iwasaki, M., et al. (2016). Pressure dynamics in the trays caused by differences of the various impression materials and thickness of the relief in the maxillary edentulous model, Journal of Prosthodontic Research

[12] Komiyama, O., et al. (2004). Effects of relief space and escape holes on pressure characteristics of maxillary edentulous impressions, The Journal of Prosthetic Dentistry, Vol. 91(6), pp. 570-576 